\title{
Os coletivos e os estilos de pensamento em pesquisas brasileiras sobre investigação-ação
}

\author{
Collectives and thinking styles in brazilian research on research-action \\ Colectivos y estilos de pensamiento en la investigación brasileña sobre \\ investigación-acción
}

Larissa Lunardi'; Rúbia Emmel²

\section{RESUMO}

Esta pesquisa analisou dissertações e teses sobre a formação inicial de professores de Ciências Biológicas, no campo da investigação-ação. Apoiou-se nas categorias epistemológicas de Fleck (2010) para compreender as contribuições dos autores-referências utilizados por dissertações e teses, no âmbito da investigação-ação, disponíveis na Biblioteca Digital Brasileira de Teses e Dissertações (BDTD). Foi possível identificar os estilos e coletivos de pensamento que caracterizam a pesquisa do tema no recorte apresentado. Com base na leitura das dissertações (5) e das teses (3), por meio da Análise Textual Discursiva (ATD), foram identificadas um total de 58 palavras-chave, as quais permitiram a elaboração de cinco agrupamentos temáticos realizados a partir da leitura e identificação de 13 autores-referências que apareceram mais de uma vez nas pesquisas sobre a temática. A constituição dos Eixos Temáticos teve papel fundamental para reconhecer as aproximações entre a base de dados da investigação-ação e as análises, demonstrando a importância da circulação de formas de pensar acerca da investigação-ação.

Palavras-chave: Formação inicial de professores; Ciências Biológicas; Ensino de Ciências; Pesquisa-ação; Epistemologia.

\begin{abstract}
This research analyzed dissertations and theses on the initial training of Biological Sciences teachers, in the field of action research. It relied on the epistemological categories of Fleck (2010) to understand the contributions of the reference authors used by dissertations and theses, within the scope of action research, available at the Brazilian Digital Library of Theses and Dissertations (BDTD). It was possible to identify the styles and collectives of thought that characterize the research of the theme in the presented section. Based on the reading of the dissertations (5) and the theses (3), through the Discursive Textual Analysis (ATD), a total of 58 keywords were identified, which allowed the elaboration of five thematic groups realized from the reading and identification of 13 reference authors who appeared more than once in research on the theme. The constitution of the Thematic Axes played a fundamental role in recognizing the similarities between the action research database and the analyzes, demonstrating the importance of circulating ways of thinking about action research.
\end{abstract}

Keywords: Initial teacher formation; Biological Sciences; Science teaching; Research-action; Epistemology.

\section{RESUMEN}

Esta investigación analizó disertaciones y tesis sobre la formación inicial del profesorado de Ciencias Biológicas, en el campo de la investigación acción. Se basó en las categorías epistemológicas de Fleck (2010) para

1 Mestra em Ensino de Ciências pela Universidade Federal da Fronteira Sul (UFFS). E-mail: larissalunardi18@gmail.com

2 Doutora em Educação nas Ciências e professora do Instituto Federal Farroupilha (IFFAR). E-mail: rubia.emmel@iffarroupilha.edu.br 
comprender los aportes de los autores de referencia utilizados por disertaciones y tesis, en el ámbito de la investigación-acción, disponibles en la Biblioteca Digital Brasileña de Tesis y Disertaciones (BDTD). Fue posible identificar los estilos y colectivos de pensamiento que caracterizan la investigación del tema en el apartado presentado. A partir de la lectura de las disertaciones (5) y las tesis (3), a través del Análisis Textual Discursivo (ATD), se identificaron un total de 58 palabras clave, lo que permitió la elaboración de cinco grupos temáticos realizados a partir de la lectura. e identificación de 13 autores de referencia que aparecieron más de una vez en investigaciones sobre el tema. La constitución de los Ejes Temáticos jugó un papel fundamental en el reconocimiento de las similitudes entre la base de datos de investigación acción y los análisis, demostrando la importancia de circular formas de pensar sobre la investigación acción.

Palabras clave: Formación docente inicial; Ciencias biologicas; Enseñanza de las ciencias; Investigaciónacción; Epistemología.

\section{INTRODUÇÃO}

Há um aumento do número de pesquisas que investigam a 'investigação-ação' e a 'formação inicial de professores de Ciências Biológicas' em programas de pós-graduação (Lima, 2010; Güllich, 2012; Souto, 2013; Canabarro, 2015; Emmel, 2015; Sobral, 2015; Dattein, 2016; Leite, 2016; Kierepka, 2017). Neste contexto, ressalta-se a investigação-ação como "uma metodologia de intervenção social cientificamente apoiada [qu]e desenrola-se segundo ciclos de planificação, ação, observação, reflexão" (Alarcão, 2011, p. 52). Ela tem por objetivo a transformação da prática docente e/ou do espaço a que se vincula essa prática, por isso sua crescente utilização em estudos referentes à formação docente (Carr; Kemmis, 1988).

Diante disso, acredita-se ser necessário investigar que concepções metodológicas são evidenciadas nas diferentes pesquisas. Por isso, este artigo apresenta uma análise interpretativa de ordem epistemológica que busca identificar, por meio de categorias epistemológicas de Fleck (2010), os estilos e os coletivos de pensamento presentes em pesquisas de dissertações e teses que utilizam a investigação-ação como método de trabalho no âmbito da formação inicial de professores de Ciências Biológicas.

A análise dessas produções é fundamental para evidenciar as concepções e caracterizações da investigação-ação, bem como os pressupostos epistemológicos e condições históricas de suas produções. Por isso, este artigo amplia os estudos sobre investigação-ação, uma vez que apoia-se nas categorias da epistemologia de Fleck (2010) para compreender as contribuições dos autoresreferências utilizados pelas publicações (teses e dissertações) cujos métodos teóricos-metodológicos se apoiam na investigação-ação.

A pesquisa de Fleck (2010), sobre esses estilos e coletivos de pensamento, foi desenvolvida a partir do princípio de que o conhecimento, nos estilos de reflexão, tem critérios que resultam em processos de análise sócio-histórico-cultural do fato em estudo (Lorenzetti; Muenchen; Slongo, 2018). Massoni e Moreira (2015) e Emmel (2011) acreditam que compreender o ponto de vista de Fleck possibilita a reflexão crítica dos professores acerca do papel da ciência e de sua natureza.

A partir desses pressupostos, foi possível (re)elaborar e propor os questionamentos: Quais eixos teóricos estão refletidos nas pesquisas sobre investigação-ação? Que estilos e coletivos de pensamento constituem estas pesquisas? É possível reconhecer os autores-referências das pesquisas sobre a investigação-ação, bem como suas contribuições, à luz da epistemologia de Fleck (2010)? 
Neste contexto, a centralidade do problema desta pesquisa é analisar como os estilos de pensamento sobre investigação-ação são formados e apresentados nas pesquisas brasileiras de formação inicial de professores de Ciências Biológicas. Assim, tem-se como objetivo compreender as contribuições dos autores mais utilizados como referências em dissertações e teses produzidas no país sobre a investigação-ação na formação inicial de professores de Ciências Biológicas, para entender como ocorre a constituição dos estilos e coletivos de pensamento que caracterizam a temática. Para tanto, foram categorizados, através da análise textual discursiva, os referenciais teóricos e diferentes concepções acerca da investigação-ação presentes na base de dados analisada.

As teses e dissertações sobre investigação-ação indexadas na Biblioteca Digital Brasileira de Teses e Dissertações (BDTD) do Instituto Brasileiro de Informação em Ciência e Tecnologia (IBICT) constituem-se como uma proposta de estudo que contém percepções, anseios, perspectivas e vozes. Esses vislumbram mudanças, na produção de conhecimentos, mais abrangentes, com várias nuances, constitutivas de estilos e coletivos de pensamento (Fleck, 2010) característicos de uma episteme sobre a investigação-ação.

Para Fleck (2010), o pensamento é social e é influenciado por fatores característicos das estruturas sociais da comunidade científica. Tais fatores apresentam uma linguagem específica, além de conhecimentos e práticas que constituem o que esse epistemólogo nomeou de estilo de pensamento. O estilo de pensamento, por sua vez, condiciona um coletivo de pensamento, ou seja, um grupo de pessoas que tem pensamentos alinhados e semelhantes entre si.

\section{METODOLOGIA}

Esta pesquisa apresenta uma abordagem qualitativa (Lüdke; André, 2001), por meio de pesquisa documental realizada pela revisão literária de trabalhos acadêmicos disponíveis na Biblioteca Digital Brasileira de Teses e Dissertações (BDTD) do Instituto Brasileiro de Informação em Ciência e Tecnologia (IBICT), no endereço eletrônico: http://bdtd.ibict.br/vufind/. O recorte de tempo para seleção de publicações realizadas foi de 2010 a 2016. Como critério de busca e delimitação do recorte de análise utilizamos os termos: Ensino de Ciências/Biologia; Formação inicial de professores de Ciências/Biologia; Pesquisa-ação e/ou Investigação-ação.

Primeiramente, com os dados obtidos na busca, foi elaborada uma matriz de apresentação, no editor de planilhas Microsoft Excel, para sistematizar informações gerais acerca das pesquisas identificadas, na qual foram descritos: autor, título, ano de publicação, instituição, palavras-chave, programa de pós-graduação e campo empírico.

Esses dados possibilitaram uma análise geral das produções publicadas no período de 2010 a 2016, registrando a existência de oito pesquisas. Foi realizada a leitura desses estudos para identificar características dessas produções, tais como o estado, a região geográfica e os referenciais utilizados.

Posteriormente, foram realizadas novas leituras a fim de identificar as referências relacionadas com a investigação-ação de cada uma das pesquisas, processo também organizado em uma planilha do Excel. Foram encontradas 39 referências considerando todas as pesquisas. Os autores que foram encontrados mais de uma vez foram selecionados e sistematizados em outro quadro, obtendo-se um total de 13 escritores. Após, foram realizadas leituras novas dirigidas com o intuito de selecionar excertos das citações diretas desses autores, o que constituiu uma nova planilha. 
Destes excertos foram criadas sínteses que explanam as principais ideias dos textos. Seguidamente, foi realizada uma leitura a partir dessas sínteses, para destacar palavras-chave que, por sua vez, foram reunidas em Eixos Temáticos com o auxílio da ferramenta de filtro do Excel. Estes Eixos Temáticos foram analisados a partir do referencial epistemológico de Fleck (2010) e possibilitaram novas inter-relações e análises que vislumbraram os coletivos e os estilos de pensamento.

A análise da pesquisa foi realizada por meio dos princípios da Análise Textual Discursiva (Moraes, 2003; Moraes; Galiazzi, 2006). Esse método envolve um processo auto-organizado de construção de metatextos, em que novos entendimentos emergem de uma sequência discursiva. A análise, aqui proposta, se estruturou a partir das seguintes etapas: unitarização - os textos elaborados por meio das interpretações e compreensões dos trabalhos foram fragmentados, tal processo possibilitou a criação de unidades de significado; categorização - foram estabelecidas relações entre essas unidades, que foram agrupadas de acordo com suas semelhanças semânticas; comunicação - foram elaborados textos descritivos e interpretativos (metatextos) sobre as categorias.

\section{PRESSUPOSTOS EPISTEMOLÓGICOS DE FLECK: OS ESTILOS E OS COLETIVOS DE PENSAMENTO}

A partir da leitura da obra "A gênese e o desenvolvimento de um fato científico", de Ludwik Fleck, traduzida no Brasil em 2009 e publicada em 2010 (pela primeira vez em 1935), foi possível identificar pontos importantes da história desse cientista e das categorias epistemológicas desenvolvidas neste artigo.

Ludwik Fleck (1896-1961) nasceu em Lwów na Polônia, sua família era de origem judaica, e logo cedo dedicou-se ao estudo na medicina. Doutorou-se em Clínica Geral na Universidade Jan Kazimierz de Lviv. Atuou como professor e como médico em inúmeras universidades, nesse contexto voltou seus esforços para o estudo da microbiologia, tendo dirigido laboratórios bacteriológicos em hospitais e institutos poloneses (Condé, 2012; Fleck, 2010).

Durante a Segunda Guerra Mundial, e com a invasão da Polônia pela Alemanha Nazista, foi enviado a um campo de concentração. Durante esse período, o Reich o designou para trabalhar na criação e confecção de vacinas contra o tifo. As experiências vivenciadas durante esse período marcaram o pesquisador, propiciando um olhar mais aprofundado sobre a natureza do pensamento científico, assim como da sociológica e histórica da ciência (Condé, 2012; Fleck, 2010).

Embora suas ideias acerca da ciência e sua história sejam extremamente relevantes, na época de publicação de seu livro em alemão "Gênese e desenvolvimento de um fato científico" (1935), foram menosprezadas (Condé, 2012). Assim, ganharam notoriedade apenas em 1979, quando finalmente foi traduzido para o inglês. A partir de então, e em menos de três décadas, suas ideias têm sido amplamente disseminadas no campo das ciências, com ênfase no campo da Educação em Ciência (Lorenzetti; Muenchen; Slongo, 2013).

Fleck (1986) formulou categorias epistemológicas, como o coletivo de pensamento. Esse coletivo é definido como "comunidade de indivíduos", quando duas ou mais pessoas "compartilham práticas, concepções, tradições e normas" (p. 27 apud Emmel, 2011, p.43) e determina um estilo de pensamento, ou seja, "um perceber dirigido com a correspondente elaboração intelectual e objetiva do percebido" (Fleck, 1986, p. 145 apudEmmel, 2011, p.43), que se caracteriza pelos problemas que interessam ao coletivo. O coletivo não deve ser confundido com um grupo fixo ou uma classe social, 
uma vez que um mesmo indivíduo pode participar de mais de um coletivo de pensamento (Fleck, 2010).

O coletivo de pensamento é constituído de um pequeno círculo esotérico e de um grande círculo exotérico. "Um indivíduo pode pertencer a vários círculos exotéricos e a uns poucos, ou nenhum, esotérico" (Fleck, 1986, p. 152 apud Emmel, 2011, p.45). O círculo esotérico é formado por especialistas de uma determinada área do conhecimento e caracteriza a identidade primeira do coletivo de pensamento, por ser a portadora do estilo de pensamento. É a partir desse núcleo de conhecimentos e de ideias compartilhadas que surge o círculo exotérico, o qual é constituído por leigos formados que passam a interagir com o círculo esotérico e adquirem o conjunto de elementos formadores do estilo de pensamento (Fleck, 2010). De acordo com Fleck (2010), esses dois círculos desenvolvem uma interdependência.

Dessa forma, neste artigo, denominamos Eixos Temáticos as categorias elaboradas a partir dos estilos de pensamento, isto é, assuntos que interessam a um coletivo de pesquisadores. Esse coletivo de pensamento é apresentado pela identificação de autores-referências que tratam acerca do tema de investigação-ação e que foram citados nas dissertações e teses pesquisadas.

\section{ANÁLISE E DISCUSSÃO: COMPREENDENDO AS DISSERTAÇÕES E TESES SOBRE INVESTIGAÇÃO-AÇÃO NA FORMAÇÃO INICIAL DE PROFESSORES EM CIÊNCIAS BIOLÓGICAS A PARTIR DOS COLETIVOS E DOS ESTILOS DE PENSAMENTO}

Considera-se, nesta pesquisa, que a análise e sistematização de pesquisas de dissertações e teses sobre a investigação-ação, com base na leitura de Fleck (2010), podem contribuir para a formação inicial de professores de Ciências Biológicas, pois evidenciam o caráter epistemológico destas pesquisas e permitem reconhecer, traçar parâmetros e socializar a produção existente sobre o tema.

Parafraseando Fleck (2010), a teoria do conhecimento não consiste somente na verificação da capacidade dos conceitos. Cabe adotar, então, uma postura mais universal, um princípio de pensamento que permita perceber um maior número de detalhes e de acoplamentos compulsórios a serem priorizados.

O Quadro 1 apresenta as dissertações e teses encontradas, e o que pode se identificar de um modo geral é que são produzidas em programas de: "Ensino de Ciências"; "Educação em/nas Ciências" ou "Educação Científica e Tecnológica". A base de dados apresentada no Quadro 1 indica um quantitativo de oito pesquisas. Foram encontrados estudos entre os anos de 2010 e 2016, sendo que o ano de 2015 (P4, P5 e P6) obteve maior número de pesquisas (três no total). P1 foi publicada no ano de 2010, P2 em 2012, P3 em 2013 e P7 e P8 em 2016.

Quadro 1 - Teses e Dissertações sobre formação inicial de professores de Ciências e Biologia com base na Investigação-ação

\begin{tabular}{|c|c|c|c|c|c|}
\hline & ANO & P & TÍTULO & IES & PPG \\
\hline P1 & 2010 & D & $\begin{array}{c}\text { Uma análise sobre a proposição de projetos } \\
\text { de trabalho com o uso das Tecnologias da } \\
\text { Informação e Comunicação (TIC) em um } \\
\text { curso de licenciatura a distância }\end{array}$ & UFRPE & Ensino das Ciências \\
\hline P2 & 2012 & T & $\begin{array}{c}\text { O livro didático, o professor e o ensino de } \\
\text { ciências: um processo de investigação- } \\
\text { formação-ação }\end{array}$ & UNIJUÍ & Educação nas Ciências \\
\hline
\end{tabular}




\begin{tabular}{|c|c|c|c|c|c|}
\hline P3 & 2013 & D & $\begin{array}{c}\text { Formação crítica mediada pelas tecnologias } \\
\text { digitais de informação e comunicação no } \\
\text { ensino de ciências: a análise de uma } \\
\text { experiência nas licenciaturas em Física e } \\
\text { Ciências Biológicas }\end{array}$ & UFSC & Educação Científica e \\
Tecnológica
\end{tabular}

Fonte: Autoras, 2019. Nota: P: Tipo de pesquisa; D: Dissertação; T: Tese; IES: Instituição de Ensino Superior; PPG: Programa de Pós-Graduação.

A partir das pesquisas dessas dissertações e teses, foram realizadas leituras e identificadas as características dessas produções, na busca por reconhecer elementos que caracterizassem os estilos de pensamento dos autores-referências. As 39 referências utilizadas sobre a investigação-ação foram tabeladas. Os autores que foram encontrados mais de uma vez foram selecionados e sistematizados em outro quadro, com o total de 13 autores. Após, foram realizadas buscas dirigidas das citações desses autores nas teses e dissertações, o que constituiu um novo quadro. Desses excertos foram destacadas palavras-chave, que foram reunidas com o auxílio da ferramenta de filtro do Excel e que constituíram os Eixos Temáticos - categorias em extensão dentro dos estilos de pensamento.

Desse modo, essas categorias/Eixos Temáticos, resultantes da pesquisa, estão expressas no Quadro 2, organizado de acordo com a ATD (Moraes; Galiazzi, 2006) a partir das palavras-chave retiradas dos excertos. A categorização foi realizada considerando a proximidade semântica e de significado das unidades.

No Quadro 2 é possível visualizar os coletivos de pensamento, que, parafraseando Fleck (2010), são grupos que, mesmo distante fisicamente, estão alinhados intelectualmente, e a produção do conhecimento, a qual segue uma determinada perspectiva epistemológica e metodológica. Esses coletivos, por sua vez, formam o estilo, ou seja, o conhecimento que parte da construção coletiva do pesquisador e seus pares, os quais usam os mesmos referenciais e tem as mesmas perspectivas. A partir das leituras em Fleck (2010) e Condé (2012), compreende-se que o olhar de cada pesquisador, suas vivências e suas histórias, contribuem para dar diferentes sentidos e visões ao mesmo objeto, no caso, a pesquisa. 
Quadro 2 - Coletivos e estilos de pensamento da Investigação-ação

\begin{tabular}{|c|c|c|c|}
\hline Eixo temático & Palavras & $\begin{array}{c}\text { Dissertação ou } \\
\text { Tese }\end{array}$ & Em quais referenciais aparece? \\
\hline \multirow{10}{*}{ Ação } & Ação & $\begin{array}{c}P 2, P 3, P 4, P 6, P 7 \\
\text { P8 }\end{array}$ & $\begin{array}{c}\text { Alarcão, I.; Carr, W. e Kemmis, S.; Contreras, J } \\
\text { D.; Rosa, M. I. P. e Schnetzler, R. P.; Barbier, } \\
\text { R.; Coutinho, C.; Güllich, R. I. C. }\end{array}$ \\
\hline & Atitude & P7 & Carr, W. e Kemmis, S. \\
\hline & Empírico & $\mathrm{P} 2, \mathrm{P} 4$ & Contreras, J. D., Thiollent, M. \\
\hline & Experiência & $\mathrm{P} 2, \mathrm{P} 4, \mathrm{P7}, \mathrm{P} 8$ & $\begin{array}{c}\text { Alarcão, I.; Carr, W. e Kemmis, S.; Contreras, J. } \\
\text { D.; Rosa, M. I. P. e Schnetzler, R. P.; Barbier, } \\
\text { R. }\end{array}$ \\
\hline & Fazer & $\mathrm{P} 1$ & Thiollent, M. \\
\hline & Intervenção & P7 & Contreras, J. D. \\
\hline & Prática & P2, P3, P6, P7, P8 & $\begin{array}{l}\text { Alarcão, I.; Carr, W. e Kemmis, S.; Contreras, J, } \\
\text { D.; Elliott, J.; Rosa, M. I. P. e Schnetzler, R. P.; } \\
\text { Tripp, D; Coutinho, C.; Güllich, R. I. C.; Person, } \\
\text { V. A. e Güllich, R. I. C.; Kierepka, J. S. N. e } \\
\text { Güllich, R. I. C.; Mion, R. A. e Saito, C. H. }\end{array}$ \\
\hline & Sistemática & $\mathrm{P} 2, \mathrm{P} 8$ & Alarcão, I.; Tripp, D. \\
\hline & Técnica & P2, P3, P7, P8 & $\begin{array}{l}\text { Carr, W. e Kemmis, S.; Contreras, J. D.; } \\
\text { Coutinho, C.; Mion, R. A. e Saito, C. H. }\end{array}$ \\
\hline & Vivências & P7 & Güllich, R. I. C. \\
\hline \multirow{18}{*}{ Social } & Associação & P4 & Thiollent, M. \\
\hline & Colaboração & P2, P3, P6, P8 & $\begin{array}{c}\text { Alarcão, I.; Carr, W. e Kemmis, S.; Contreras, J } \\
\text { D.; Elliott, J.; Rosa, M. I. P. e Schnetzler, R. P.; } \\
\text { Tripp, D; Coutinho, C. }\end{array}$ \\
\hline & Coletivo & $\mathrm{P} 2, \mathrm{P} 4, \mathrm{P} 7$ & $\begin{array}{l}\text { Thiollent, M.; Contreras, J. D.; Barbier, R.; } \\
\text { Güllich, R. I. C. }\end{array}$ \\
\hline & Compartilhado & $\mathrm{P} 2$ & Tripp, D. \\
\hline & $\begin{array}{l}\text { Comunidades } \\
\text { autorreflexivas }\end{array}$ & P2 & Carr, W. e Kemmis, S. \\
\hline & Contexto escolar & P7 & Person, V. A. e Güllich, R. I. C. \\
\hline & Cooperativo & $\mathrm{P} 2, \mathrm{P} 4$ & Contreras, J. D.; Thiollent, M \\
\hline & Democrática & P7, P8 & Alarcão, I.; Carr e Kemmis, S. \\
\hline & Diálogo & P2, P3, P7, P8 & Alarcão, I.; Coutinho, C.; Güllich, R. I. C. \\
\hline & Histórico & $\mathrm{P} 2, \mathrm{P} 7, \mathrm{P} 8$ & Carr, W. e Kemmis, S. \\
\hline & Indivíduo & $\mathrm{P} 2, \mathrm{P} 7, \mathrm{P} 8$ & Contreras, J. D.; Carr, W. e Kemmis, S. \\
\hline & Integralidade & P4 & Barbier, R. \\
\hline & Interação & $\mathrm{P} 2, \mathrm{P} 7, \mathrm{P} 8$ & Carr, W. e Kemmis, S.; Tripp, D. \\
\hline & Interdependência & P3 & Coutinho, C. \\
\hline & Participação & P2, P3, P4, P7, P8 & $\begin{array}{l}\text { Thiollent, M.; Alarcão, I.; Carr, W. e Kemmis, } \\
\text { S.; Barbier, R.; Coutinho, C.; Güllich, R. I. C. }\end{array}$ \\
\hline & Pesquisa social & P4 & Thiollent, $\mathrm{M}$. \\
\hline & Social & P2, P3, P4, P7, P8 & $\begin{array}{c}\text { Carr, W. e Kemmis, S.; Contreras, J. D.; } \\
\text { Barbier, R.; Coutinho, C. }\end{array}$ \\
\hline & Sociedade & $\mathrm{P} 2, \mathrm{P} 8$ & Carr, W. e Kemmis, S. \\
\hline \multirow{2}{*}{ Transformação } & Autonomia & $\mathrm{P} 2, \mathrm{P} 7, \mathrm{P} 8$ & Alarcão, I.; Contreras, J. D.; Güllich, R. I. C. \\
\hline & Conflito & P7 & Carr, W. e Kemmis, S. \\
\hline
\end{tabular}




\begin{tabular}{|c|c|c|c|}
\hline & Criativo & P7, P8 & Alarcão, I. \\
\hline & Dialética & P7, P8 & Carr, W. e Kemmis, S. \\
\hline & Emancipatória & P3, P7, P8 & $\begin{array}{l}\text { Carr, W. e Kemmis, S.; Contreras, J. D.; } \\
\text { Coutinho, C.; Güllich, R. I. C. }\end{array}$ \\
\hline & Mudança & P2, P7, P8 & $\begin{array}{c}\text { Alarcão, I.; Carr, W. e Kemmis, S.; Coutinho, } \\
\text { C.; Güllich, R. I. C. }\end{array}$ \\
\hline & Práxis & $\mathrm{P} 2, \mathrm{P} 4, \mathrm{P} 7, \mathrm{P} 8$ & $\begin{array}{c}\text { Carr, W. e Kemmis, S.; Contreras, J. D.; } \\
\text { Barbier, R. }\end{array}$ \\
\hline & Transformação & P2, P3, P6, P7, P8 & $\begin{array}{c}\text { Alarcão, I.; Carr, W. e Kemmis, S.; Contreras, J. } \\
\text { D.; Elliott, J.; Rosa, M. I. P. e Schnetzler, R. P.; } \\
\text { Coutinho, C.; Güllich, R. I. C.; Person, V. A. e } \\
\text { Güllich, R. I. C. }\end{array}$ \\
\hline \multirow{11}{*}{ Formação } & Concepções & P7 & Carr, W. e Kemmis, S. \\
\hline & Conhecimento & $\mathrm{P} 2, \mathrm{P} 3, \mathrm{P} 7, \mathrm{P} 8$ & $\begin{array}{l}\text { Alarcão, I.; Carr e Kemmis, S.; Mion, R. A. e } \\
\text { Saito, C. H. }\end{array}$ \\
\hline & Construção & $\mathrm{P} 2, \mathrm{P} 7$ & Alarcão, I.; Contreras, J. D. \\
\hline & Desenvolvimento & $\mathrm{P} 2, \mathrm{P} 6, \mathrm{P} 8$ & $\begin{array}{c}\text { Alarcão, I.; Carr, W. e Kemmis, S.; Elliott, J.; } \\
\text { Rosa, M. I. P. e Schnetzler, R. P.; Güllich, R. I. } \\
\text { C. }\end{array}$ \\
\hline & Diário de bordo & $\mathrm{P} 2, \mathrm{P} 8$ & $\begin{array}{l}\text { Rosa, M. I. P. e Schnetzler, R. P.; Güllich, R. I. } \\
\text { C.; Kierepka, J. S. N. e Güllich, R. I. C. }\end{array}$ \\
\hline & Discurso & P6, P7 & Elliott, J.; Güllich, R. I. C. \\
\hline & Formação & $\mathrm{P} 2, \mathrm{P} 6, \mathrm{P} 7, \mathrm{P} 8$ & $\begin{array}{l}\text { Alarcão, I.; Contreras, J. D.; Rosa, M. I. P. e } \\
\text { Schnetzler, R. P.; Tripp, D.; Güllich, R. I. C.; } \\
\text { Person, V. A. e Güllich, R. I. C.; Kierepka, J. S. } \\
\text { N. e Güllich, R. I. C.; Mion, R. A. e Saito, C. H. }\end{array}$ \\
\hline & Narrativas & $\mathrm{P} 2, \mathrm{P} 8$ & $\begin{array}{l}\text { Alarcão, I.; Contreras, J. D.; Rosa, M. I. P. e } \\
\text { Schnetzler, R. P.; Güllich, R. I. C.; Kierepka, J. } \\
\text { S. N. e Güllich, R. I. C. }\end{array}$ \\
\hline & Processo & P2, P3, P6, P7, P8 & $\begin{array}{c}\text { Alarcão, I.; Carr, W. e Kemmis, S.; Contreras, J. } \\
\text { D.; Elliott, J.; Rosa, M. I. P. e Schnetzler, R. P.; } \\
\text { Coutinho, C.; Güllich, R. I. C.; Person, V. A. e } \\
\text { Güllich, R. I. C.; Kierepka, J. S. N. e Güllich, R. } \\
\text { I. C. }\end{array}$ \\
\hline & Significado & P7, P8 & $\begin{array}{c}\text { Alarcão, I.; Carr, W. e Kemmis, S.; Kierepka, J. } \\
\text { S. N. e Güllich, R. I. C. }\end{array}$ \\
\hline & Teoria & $\mathrm{P} 2, \mathrm{P} 6, \mathrm{P7}, \mathrm{P} 8$ & $\begin{array}{c}\text { Carr, W. e Kemmis, S.; Contreras, J. D.; Güllich, } \\
\text { R. I. C. }\end{array}$ \\
\hline \multirow{7}{*}{ Reflexão } & Aprimorar & $\mathrm{P} 2$ & Tripp, D. \\
\hline & Cíclico & $\mathrm{P} 2, \mathrm{P} 3, \mathrm{P} 6$ & Alarcão, I.; Coutinho, C. \\
\hline & Crítica & $\mathrm{P} 2, \mathrm{P} 3, \mathrm{P} 7, \mathrm{P} 8$ & $\begin{array}{l}\text { Carr, W. e Kemmis, S.; Contreras, J. D.; Elliott, } \\
\text { J.; Coutinho, C.; Güllich, R. I. C.; Mion, R. A. e } \\
\text { Saito, C. H. }\end{array}$ \\
\hline & Investigação & P2, P3, P6, P7, P8 & $\begin{array}{l}\text { Carr, W. e Kemmis, S.; Contreras, J. D.; } \\
\text { Coutinho, C.; Güllich, R. I. C. }\end{array}$ \\
\hline & Observação & $\mathrm{P} 2, \mathrm{P} 3, \mathrm{P} 6$ & Alarcão, I.; Carr, W. e Kemmis, S. \\
\hline & Pensamento & $\mathrm{P} 2, \mathrm{P} 7, \mathrm{P} 8$ & Carr, W. e Kemmis, S.; Contreras, J. D. \\
\hline & Planejamento & P2, P3, P6, P8 & $\begin{array}{c}\text { Alarcão, I.; Carr, W. e Kemmis, S.; Contreras, J. } \\
\text { D. }\end{array}$ \\
\hline
\end{tabular}




\begin{tabular}{|c|c|c|c|}
\hline & Problema & P4, P8 & Thiollent, M.; Carr, W. e Kemmis, S. \\
\cline { 2 - 4 } & Questionamentos & P8 & Alarcão, I.; Carr, W. e Kemmis, S. \\
\cline { 2 - 4 } & Racionalidade & P7, P8 & Carr, W. e Kemmis, S.; Contreras, J. D. \\
\cline { 2 - 4 } & & $\begin{array}{c}\text { Alarcão, I.; Carr, W. e Kemmis, S.; Contreras, J. } \\
\text { D.; Elliott, J.; Coutinho, C.; Güllich, R. I. C.; } \\
\text { Person, V. A. e Güllich, R. I. C.; Kierepka, J. S. } \\
\text { N. e Güllich, R. I. C. }\end{array}$ \\
\hline
\end{tabular}

Fonte: Autoras, 2019.

Foram identificados um total de 39 autores-referências utilizados teoricamente para dissertar acerca da investigação-ação nas teses e dissertações. Os 13 autores que aparecem mais de uma vez são: Alarcão (1996, 2000, 2001, 2010, 2011); Carr e Kemmis (1988); Contreras (1994, 2002); Rosa e Schnetzler (2003); Barbier (2002); Coutinho (2008, 2009); Güllich (2004, 2009, 2010, 2012, 2013); Tripp (2005); Elliott (1990, 1998); Kierepka e Güllich (2013); Mion e Saito (2001); Person e Güllich (2015); e Thiollent (1997, 1988, 2004). Destaca-se que Carr e Kemmis, e Contreras foram encontrados em cinco pesquisas, ou seja, são os autores mais utilizados para referenciar a investigação-ação.

Durante a ATD foram encontradas 58 palavras-chave, as quais foram reorganizadas por temas. Essa reorganização originou os fragmentos que permitiram constituir um patchwork, evidenciando as relações entre autores das pesquisas, autores-referências e temas de pesquisa. A definição conceitual dos eixos temáticos fundamentou essa constituição, a qual, por sua vez, delineou e originou os cinco Eixos Temáticos que são apresentados e analisados a seguir.

Os autores-referências, cujas citações identificaram mais palavras, estão presentes no Gráfico 1. Já sobre os demais autores-referências (Thiollent, M.; Elliott, J.; Barbier, R.; Tripp, D; Kierepka, J. S. N. e Güllich, R. I. C.; Person, V. A. e Güllich, R. I. C.; Mion, R. A. e Saito, C. H.) foram encontradas menos de 10 palavras-chave.

Gráfico 1 - Número de palavras identificadas por autores-referências

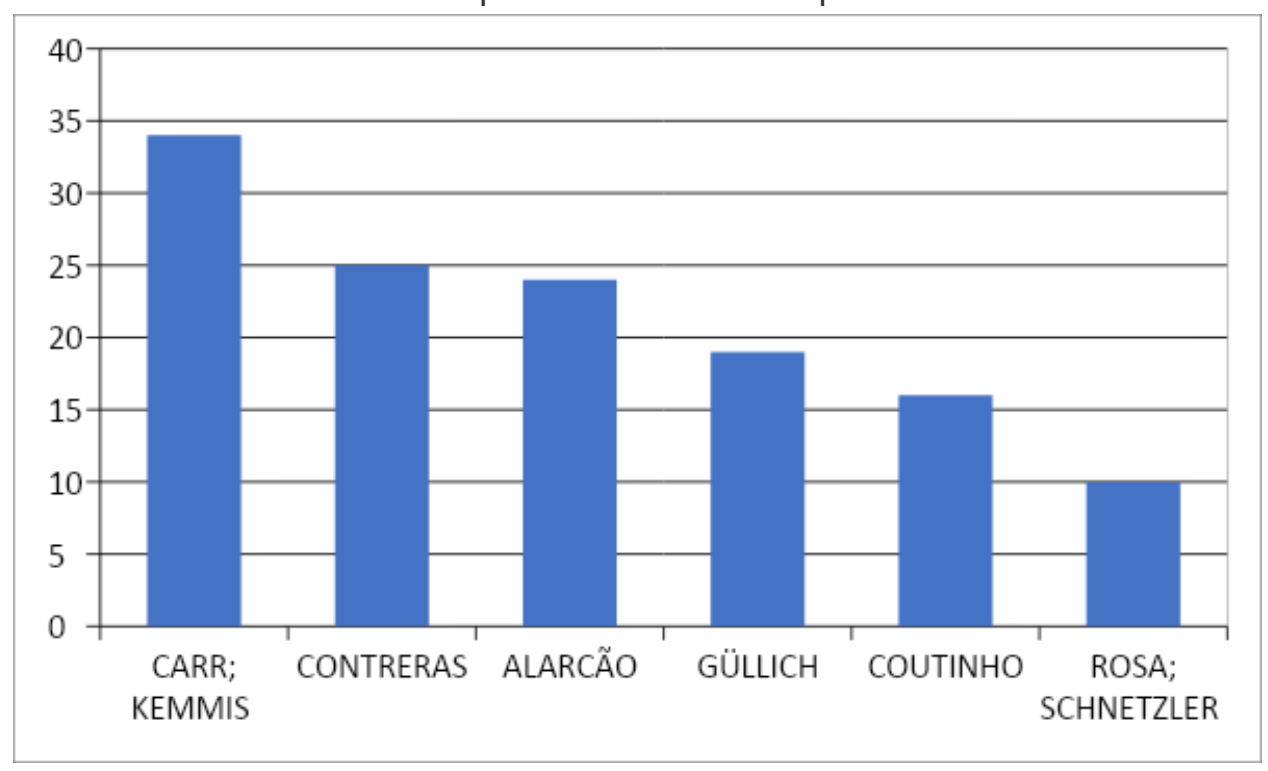

Fonte: Autoras (2019)

Os agrupamentos temáticos acerca da investigação-ação foram realizados para reconhecer os estilos e coletivos de pensamento. Essa constituição se fez a partir da vontade de fortalecer a formação inicial de professores de Ciências, na perspectiva de melhor compreender suas teorizações, seus 
conhecimentos, o que possibilitou rever conceitos. Foram identificados um total de cinco Eixos Temáticos (estilos de pensamento). O Eixo Temático que possui mais palavras-chave é "Social", com 18.

Desse modo, foi possível perceber a circulação de ideias dos autores-referências em diferentes Eixos Temáticos. Os autores-referências Alarcão, I.; Carr, W e Kemmis, S.; Contreras, J. D.; Coutinho, C.; Güllich, R. I. C.; Elliott, J.; Person, V. A. e Güllich, R. I. C. foram identificados em todos os Eixos Temáticos, por exemplo.

O Eixo Temático "Social" é formado pelas palavras: associação, colaboração, coletivo, compartilhado, comunidades autorreflexivas, contexto escolar, cooperativo, democrática, diálogo, histórico, indivíduo, integralidade, interação, interdependência, participação, pesquisa social, social e sociedade. Essas palavras foram identificadas em citações de 11 autores e em seis pesquisas (P2, P3, P4, P6, P7 e P8). A investigação-ação pode ser tratada como uma pesquisa social que busca a resolução de um problema coletivo (Thiollent, 1988). De acordo com Carr e Kemmis (1988), o pensamento e a ação individuais passam a ter sentido em um contexto social e histórico.

O Eixo Temático "Ação" é formado pelas palavras: ação, atitude, empírico, experiência, fazer, intervenção, prática, sistemática, técnica, vivências. As palavras evidenciaram-se em citações de 13 autores e em sete pesquisas (P1, P2, P3, P4, P6, P7 e P8). Segundo Contreras (1994), a investigaçãoação integra o conhecimento e a ação, já que é necessário conhecer e atuar no processo exploratório para converter a prática em objeto de investigação.

O Eixo Temático "Formação" é formado pelas palavras: concepções, conhecimento, construção, desenvolvimento, diário de bordo, discurso, formação, narrativas, processo, significado, teoria. Essas palavras foram encontradas em citações de 11 autores e em cinco pesquisas (P2, P3, P6, P7 e P8). Conforme Güllich (2012), a formação de professores, a partir da investigação-ação, possibilita o desenvolvimento de maneira coletiva, proporcionando um processo integral assumido pelos participantes.

O Eixo Temático "Reflexão" é formado pelas palavras: aprimorar, cíclico, crítica, investigação, observação, pensamento, planejamento, problema, questionamentos, racionalidade, reflexão. Essas palavras aparecem nas citações de 11 autores e em seis pesquisas (P2, P3, P4, P6, P7 e P8). De acordo com Alarcão (2010), a experiência é analisada e conceituada por meio de processos de observação e reflexão, sendo que esse processo resulta na transformação, que guia outra experiência e, assim, reinicia-se o ciclo.

O Eixo Temático "Transformação" é formado pelas palavras: autonomia, dialética, emancipatória, mudança, práxis, transformação. As palavras foram identificadas em citações de nove autores e em seis pesquisas (P2, P3, P4, P6, P7 e P8). A investigação-ação tem como objetivo a transformação de práticas e a sua consequente melhoria gradativa e colaborativa (Contreras, 1994). Essa transformação é social e, para isso, requer a participação de todos os envolvidos na prática (Coutinho, 2009).

Os Eixos Temáticos aqui expostos permitiram reconhecer que há uma aproximação entre as pesquisas da investigação-ação, firmando a existência da circulação de ideias (Fleck, 2010) entre as pesquisas e revelando as suas interfaces e nuances. A circulação de ideias, que se apresentou na identificação dos Eixos Temáticos, permitiu identificar formas mais amplas de constituir e de pensar a investigaçãoação. 


\section{A EPISTEMOLOGIA FLECKIANA COMO PERSPECTIVA DE RECONHECIMENTO DE COLETIVOS DE PENSAMENTO NO CONTEXTO DA INVESTIGAÇÃO-AÇÃO NA FORMAÇÃO DE PROFESSORES EM CIÊNCIAS BIOLÓGICAS}

Todo o processo de constituição de matrizes (quadros e tabelas) permitiu uma imersão em cada dissertação ou tese, bem como em cada autor/a e referência. Nesse sentido, o Quadro 3 demonstra essas informações e permite elucidar os coletivos de pensamento que se desenrolam nos ciclos da investigação-ação.

Quadro 3 - Coletivos de pensamento formados pelos autores-referências dos ciclos de investigação-ação

\begin{tabular}{|c|c|c|c|}
\hline $\begin{array}{c}\text { Eixo } \\
\text { temático }\end{array}$ & Palavras & $\begin{array}{c}\text { Dissertação } \\
\text { ou Tese }\end{array}$ & Em quais referenciais aparece? \\
\hline \multirow{7yyy}{*}{ Espiral/ciclo } & Observação & P2, P3, P6 & Alarcão, I.; Carr, W. e Kemmis, S. \\
\cline { 2 - 4 } & Reflexão & $\begin{array}{c}\text { P2, P3, P6, } \\
\text { P7, P8 }\end{array}$ & $\begin{array}{c}\text { Alarcão, I.; Carr, W. e Kemmis, S.; Contreras, J. D.; Elliott, } \\
\text { J.; Coutinho, C.; Güllich, R. I. C.; Person, V. A. e Güllich, R. } \\
\text { I. C.; Kierepka, J. S. N. e Güllich, R. I. C. }\end{array}$ \\
\cline { 2 - 5 } & $\begin{array}{c}\text { Planejament } \\
\text { O P2, P3, P6, }\end{array}$ & Plarcão, I.; Carr, W. e Kemmis, S.; Contreras, J. D. \\
\cline { 2 - 4 } & Ação & $\begin{array}{c}\text { P2, P3, P4, } \\
\text { P6, P7, P8 }\end{array}$ & $\begin{array}{r}\text { Alarcão, I.; Carr, W. e Kemmis, S.; Contreras, J. D.; Rosa, } \\
\text { M. I. P. e Schnetzler, R. P.; Barbier, R.; Coutinho, C.; } \\
\text { Güllich, R. I. C. }\end{array}$ \\
\hline
\end{tabular}

Fonte: Autores, 2019.

Foi possível evidenciar um total de 10 autores-referência utilizados para exemplificar o desenvolvimento dos ciclos de investigação-ação nas teses e dissertações encontradas. A palavra do ciclo que apresenta mais autores-referência é "reflexão" (oito autores). As palavras do ciclo foram evidenciadas em seis pesquisas, sendo três dissertações e três teses: observação (três pesquisas), reflexão (cinco pesquisas), planejamento (quatro pesquisas) e ação (seis pesquisas).

O ciclo apresentado no quadro é utilizado por alguns autores, como Alarcão (2011), que aborda a investigação-ação como "uma metodologia de intervenção social cientificamente apoiada e desenrolase segundo ciclos de planificação, ação, observação, reflexão" (p. 52). Com isso, observou-se que autores como Carr e Kemmis e Alarcão apresentaram, em suas citações, todas as palavras do ciclo, ou seja, acreditam que a investigação-ação seja desenvolvida a partir do planejamento, seguido pela ação, observação e reflexão, processos que resultam em um novo planejamento, reiniciando o ciclo.

Porém, também foi possível perceber que, por mais que não tenhamos identificados em outros autores todas as palavras do ciclo, há uma circulação das ideias entre os autores-referências, sendo que apenas três (Tripp, D; Mion, R. A. e Saito, C. H.; Thiollent, M.) não apresentaram nenhuma das palavras do ciclo. Isso significa que há influências e trocas de ideias entre os próprios autoresreferências. Assim, se inspiraram em perspectivas outras, de outros autores, mas adicionaram suas próprias experiências para (re)construir novas concepções acerca da investigação-ação.

As citações dos autores-referências em que foram identificadas palavras pertencentes ao eixo temático "Espiral/ciclo" constituem o coletivo de pensamento que, no processo de investigação-ação, defende o ciclo de planejamento, ação, observação e reflexão. Esses autores-referências constituem 
o círculo esotérico que forma um núcleo de conhecimentos contribuintes para o compartilhamento das ideias entre os autores cujas pesquisas se inserem no círculo exotérico (Fleck, 2010).

O círculo esotérico é formado por especialistas de uma determinada área do conhecimento e caracteriza a identidade primeira do coletivo de pensamento, por ser o portador do estilo de pensamento. É desse núcleo de conhecimentos e de ideias compartilhadas por ele que deriva o círculo exotérico, constituído por leigos formados que passam a interagir com o círculo esotérico, e adquirem o conjunto de elementos formadores do estilo de pensamento (Fleck, 2010).

\section{CONSIDERAÇÕES FINAIS}

O estudo teve como intuito identificar teóricos, denominados nesta pesquisa como autoresreferências, utilizados em teses e dissertações que tratam acerca da investigação-ação. Assim, buscou-se ressaltar quantas vezes esses autores-referências são citados e como constituem, ou não, os estilos e os coletivos de pensamento; bem como buscou-se elucidar, por meio da ATD, os Eixos Temáticos categorizados. Os objetivos estabelecidos nesta pesquisa foram, de alguma forma, respondidos. Por isso, enfatiza-se a necessidade de identificar as pesquisas sobre investigação-ação, segundo sua organização interna, autores e referências, uma vez que, apesar das inúmeras pesquisas, pouco se sabe sobre as relações e diálogos entre as diferentes concepções.

Esta pesquisa almejou contribuir, desse modo, com o debate em torno das características da investigação-ação, de modo que pudesse apontar tanto as tendências que estão se delineando no entorno deste recorte temático, como contribuir para novas pesquisas desenvolvidas. Nesse sentido, com base nos dados coletados, foi possível perceber que os autores: Carr e Kemmis; Contreras, foram os mais utilizados para referenciar essa metodologia; eles foram citados em cinco das oito pesquisas analisadas. Assim, a importância de produzir conhecimentos, tornando-os mais abrangentes e dando a eles outras nuances, é reconhecida à ação e seus estilos, visto que produz coletivos de pensamento que perpassam, rompem ou, ao menos, propõem uma episteme para os pesquisadores da investigação-ação no recorte que apresentamos.

Com relação à constituição dos Eixos Temáticos (estilos de pensamentos) analisados, é possível compreender que tiveram papel fundamental para reconhecer as aproximações entre as bases de dados da investigação-ação, posto que as análises demonstraram a importância da circulação de ideias e permitiram compreender as principais concepções da investigação-ação. Ademais, foram importantes para a construção de uma nova compreensão sobre o tema, de modo a fortalecê-lo na perspectiva da formação inicial de professores, suas teorizações e conhecimentos.

Não obstante, importa salientar que também há a possibilidade de ir além de referenciais consagrados na investigação-ação, por meio da realização e divulgação de pesquisas que ampliem a circulação de ideias e permitam (re)atualizar os conceitos. Assim, verifica-se a necessidade de novas pesquisas de cunho epistemológico sobre a investigação-ação, para que seja possível estabelecer consciência das complicações no estilo de pensamento dos pesquisadores sobre o tema e as suas consequentes transformações para as práticas dos leitores das pesquisas, como professores e pesquisadores. 


\section{REFERÊNCIAS}

ALARCÃO, I. Escola reflexiva e nova racionalidade. Porto Alegre: Artmed, 2001.

ALARCÃO, I. (Org.) Formação reflexiva de professores: estratégias de supervisão. Porto: Porto Editora, 1996.

ALARCÃO, I. Professores reflexivos em uma escola reflexiva. 7 ed. São Paulo: Cortez, 2010.

ALARCÃO, I. Professores reflexivos em uma escola reflexiva. 8 ed. São Paulo: Cortez, 2011.

ALARCÃO, I. Ser professor reflexivo. In: ALARCÃO, I. Formação Reflexiva. Estratégias de Supervisão. Porto Editora: Porto, 2000.

BARBIER, R. A pesquisa-ação. Tradução por LucieDidio. Brasília: Plano, 2002. Série Pesquisa em Educação, v.3.

CANABARRO, P. H. O. A contribuição do PIBID na formação de professores de Biologia : uma reflexão sobre a prática (Dissertação). Brasília: UnB, 2015.

CARR, W; KEMMIS, S. Teoria Crítica de la enseñanza - la investigación- acción em la formación del profesorado. Barcelona: Martinez Rocca. 1988.

CONDÉ, M. L. L. (org.). Ludwik Fleck: estilos de pensamento na ciência. Belo Horizonte: Fino Traço, 2012.

CONTRERAS, J. D. Autonomia dos professores. São Paulo: Cortez, 2002.

CONTRERAS, J. D. La investigación en la acción. Cuadernos de Pedagogia, n 224, Madrid: Morata, abril 1994, p. 7-31.

COUTINHO, C. Investigação-Ação: metodologia preferencial nas práticas educativas. Universidade do Minho, 2008.

COUTINHO, C. P. et al. Investigacao-accao: metodologia preferencial nas praticas educativas. Psicologia, Educação e Cultura, Portugal, v. XIII No 2, p. 355-379, 2009.

DATTEIN, R. W. A mediação de escritas reflexivas compartilhadas na formação em ciências no contexto de um processo de iniciação à docência (Dissertação). Ijuí: Unijuí, 2016.

ELLIOTT, J. La investigación-acción en educación. Madrid: Ediciones Morata, 1990.

ELLIOTT, J. Recolocando a Pesquisa-ação em seu lugar original e próprio. In: GERALDI, C.; FIORENTINI, D.; PEREIRA, E. (orgs.). Cartografias do trabalho docente: professor(a) pesquisador(a). Campinas: Mercado de letras, 1998. p.137-152.

EMMEL, R. O currículo e o livro didático da Educação Básica: contribuições para a formação do licenciando em Ciências Biológicas (Tese). Ijuí: Unijuí, 2015.

EMMEL, R. "Estado da arte" e coletivos de pensamento da pesquisa sobre o livro didático no Brasil (Dissertação). Ijuí: Unijuí, 2011.

FLECK, L. La gênesis y desarrollo de um hecho científico. Tradução de Luis Meana. Madrid: Alianza Editorial, 1986. 
FLECK, L. Gênese e desenvolvimento de um fato científico. Tradução de Georg Otte e Mariana Camilo de Oliveira. Belo Horizonte: Fabrefactum, 2010.

GÜLLICH, R. I. C. Desconstruindo a imagem do livro didático no ensino de ciências. Revista SETREM. Três de Maio, v. 4, n. 3, p. 43 - 51, jan. 2004.

GÜLLICH, R. I. C. Investigação-formação-ação em ciências: um caminho para reconstruir a relação entre livro didático, o professor e o ensino. Curitiba: Prismas, 2013.

GÜLLICH, R. I. C. O livro didático, o professor e o ensino de ciências: um processo de investigaçãoformação-ação (Tese). Ijuí: Unijuí, 2012.

GÜLLICH, R. I. C.; EMMEL, R.; PANSERA-DE-ARAÚJO, M. C. Interfaces da Pesquisa sobre o Livro Didático de Ciências. In: Caderno de Resumos do VII Encontro Nacional de Pesquisa em Educação em Ciências. Florianópolis: Abrapec, 2009.

GÜLLICH, R. I. C.; PANSERA-DE-ARAÚJO, M. C.; EMMEL, R. Contribuições dos Artigos Brasileiros Indexados no Scielo sobre a Pesquisa acerca do Livro Didático no Brasil: 1999-2008. In: Anais do VIII Encontro de Pesquisa em Educação da Região Sul, ANPED SUL. Londrina: UEL, 2010.

GÜLLICH, R. I. C.; PANSERA-DE-ARAÚJO, M. C.; EMMEL, R. Interfaces da pesquisa sobre o livro didático de ciências no ensino fundamental. In: Encontro Nacional de Didática e Prática de Ensino, XV, 2010, Belo Horizonte. Anais... Belo Horizonte: UFMG, 2010.

KIEREPKA, J. S. N.; GÜLLICH, R. I. C. O papel da reflexão na constituição docente: investigação-ação como processo de intervenção. In: Anais do VI Encontro Regional Sul de Ensino de Biologia (EREBIO-SUL) e XVI Semana Acadêmica de Ciências Biológicas. URI - Santo Ângelo/RS, 2013.

KIEREPKA, J. S. N. Problematização e reconhecimento de teorias e práticas de professores em formação para o ensino de ciências com foco no educar pela pesquisa (Dissertação). Ijuí: Unijuí, 2017.

LEITE, F. A. Desenvolvimento do coletivo de pensamento da área de ensino de ciências da natureza e suas tecnologias em processos de formação de professores (Tese). Ijuí: Unijuí, 2016.

LIMA, L. F. Uma análise sobre a proposição de projetos de trabalho com o uso das Tecnologias da Informação e Comunicação (TIC) em um curso de licenciatura a distância (Dissertação). Recife: Universidade Federal Rural de Pernambuco, 2010.

LORENZETTI, L.; MUENCHEN, C.; SLONGO, I. I. P. Revista Brasileira de Ensino de Ciência e Tecnologia, Ponta Grossa, v. 11, n. 1, p. 373-404, jan./abr. 2018.

LÜDKE, M.; ANDRÉ, M. E. D. A. Pesquisa em educação: abordagens qualitativas. São Paulo: Epu, 2001.

MASSONI, N. T.; MOREIRA, M. A. A Epistemologia de Fleck: Uma Contribuição ao Debate sobre a Natureza da Ciência. Alexandria Revista de Educação em Ciência e Tecnologia, v.8, n.1, p.237264, maio 2015.

MION, R. A.; SAITO, C. H. Investigação-Ação: mudando o trabalho de formar professores. Ponta Grossa: Gráfica Planeta, 2001.

MORAES, R. Uma tempestade de luz: a compreensão possibilitada pela análise textual discursiva. Bauru: Ciência e Educação, v.9, n. 2, p. 191-211, 2003. 
MORAES, R.; GALIAZZI, M. C. Análise textual discursiva: processo reconstrutivo de múltiplas faces. Bauru: Ciência e Educação, v. 12, n. 1, p. 117-128, 2006.

PERSON, V. A.; GÜLLICH, R. I. C. Investigação-ação: da extensão à análise de processos formativos de professores de ciências. In: BONOTTO, D. L.; SANTOS, E. G.; WENZEL, J. S. (orgs). Movimentos Formativos: caminhos e perspectivas na formação de professores. Cerro Largo/RS: Polimpressos Serviços Gráficos LTDA, 2015.

ROSA, M. I. P. SCHNETZLER, R. P. A investigação-ação na formação continuada de professores de Ciências. Ciência e Educação. v. 9, n. 1, p. 27-39, 2003.

SOBRAL, M. S. C. Relevância dos laboratórios de aulas práticas na formação inicial de professores de ciências e biologia (Dissertação). Porto Alegre: UFRGS, 2015.

SOUTO, I. N. Formação crítica mediada pelas tecnologias digitais de informação e comunicação no ensino de ciências: a análise de uma experiência nas licenciaturas em Física e Ciências (Dissertação). Florianópolis: UFSC, 2013.

THIOLLENT, M. A metodologia da pesquisa-ação. São Paulo: Cortez, 2004.

THIOLLENT, M. Metodologia da pesquisa-ação. 4 ed. São Paulo: Cortez: autores associados, 1988.

THIOLLENT, M. Pesquisa-ação nas organizações. São Paulo: Atlas, 1997.

TRIPP, D. Pesquisa-ação: uma introdução metodológica. Educação e Pesquisa. v. 31, n. 3, p. 443446, 2005.

Submissão: 20/11/2020

Aceito: 15/01/2021 